\title{
Case study of Relaxation and Counterconditioning Therapy for Misophonia: A Conditioned Aversive Reflex Disorder
}

\author{
Accepted for publication, October 8, 2021, by Psychological Thought \\ Preprint
}

Thomas H Dozier ${ }^{a}$

[a] Misophonia Institute, Livermore, CA, USA

\begin{abstract}
Misophonia is an under-studied condition in which a person has intense emotional and physiological reactions to subtle stimuli (e.g., chewing sounds or hair twirling) which cannot be classified using DSM-5 criteria. This case illustrates the conceptualization of misophonia as a conditioned aversive reflex disorder consisting of a physical (e.g., muscle) reflex elicited by the misophonic trigger stimulus and subsequent emotional, physiological, and behavioral responses. This case describes a successful behavioral treatment of a middle-aged woman who was disabled by severe misophonia. The treatment included identifying the initial physical reflex, progressive muscle relaxation, and counterconditioning the initial physical reflex. Counterconditioning was accomplished by relaxing the initial physical reflex muscle during exposure to in vivo trigger stimuli, while using ambient sound as needed to reduce the severity of the misophonic response. The overall severity of misophonia reduced over the course of the 13-week treatment, based on client self-report. Data were analyzed using recovery percentage formula. The recovery percentage average of 3 scales was $82.1 \%$ at end of treatment and $93.1 \%$ at 1 -year follow-up. In this case, when the initial physical reflex's muscle was held relaxed by the patient when exposed to trigger stimuli, the initial physical reflex and the emotional reflex diminished and extinguished. This theory of misophonia, as a conditioned physical reflex to subtle stimuli, should be an essential consideration for research of the etiology, expansion, maintenance, and treatment of misophonia.
\end{abstract}

Keyword: misophonia, anger, counterconditioning, case study, Pavlovian conditioning, treatment, progressive muscle relaxation

\section{Introduction}

Misophonia is an under-studied condition in which a person has intense emotional and physiological reaction to subtle stimuli (e.g., chewing sounds or hair twirling). The median age of onset is in late childhood, but misophonia can develop at any age (Claiborn et al., 2020). Misophonia likely affects more than $10 \%$ of adults in the United States and is more prevalent in clinical populations. Studies reported $13.5 \%$ prevalence in a community sample (Cash, 2015), 19.9\% and $18.4 \%$ in undergraduate psychology students (Wu et al., 2014, Cash, 2015), 20.3\% in females living in university dormitories (Roushani \& Honarmand, 2021), and $35.0 \%$ and $66.7 \%$ in clinical populations ( Ferrer-Torres, \& Giménez-Llort, 2021; Quek et al., 2018). Misophonia is a chronic condition without proven treatment options. 


\subsection{Misophonia Treatment}

There are no empirically validated treatments for misophonia. Exposure to misophonic triggers often increase misophonia severity (Dozier, 2015b; Ferrer-Torres, \& Giménez-Llort, 2021); and a pilot study found mere exposure to trigger stimuli did not decrease the misophonic response, rather it usually increased it (Schröder et al., 2017). Several case studies reported varied positive response to treatment with cognitive behavioral therapy (McGuire et al., 2015; Reid et al., 2016; Roushani \& Honarmand, 2021) dialectical behavioral therapy (Kamody \& Del Conte, 2017), and mindfulness and acceptance (Schneider and Arch, 2017). A primary goal of these treatments was to tolerate misophonic triggers with reduced distress and not exhibit avoidant or aggressive behavior. Two counterconditioning studies (Dozier, 2015a, 2015c) reported positive response to treatment with a goal of reducing the acquired reflex responses to misophonic triggers.

One study reported treatment of 184 misophonia patients using tinnitus retraining therapy (Jastreboff \& Jastreboff, 2014). This treatment included masking with ear-level sound generators, exposure to varying levels of positive and neutral sound, and counterconditioning using very positive sounds mixed with increasing levels of misophonic sounds. Based on threshold of a 2-point reduction on a 10 -point self-report scale, $83 \%$ exhibited positive response to treatment.

There have been two controlled CBT treatment studies by associates at the University of Amsterdam (Jager et al., 2020; Schröder et al., 2017). The first study used task concentration exercises, counterconditioning, stimulus manipulation, and relaxation exercises and reported $48 \%$ of participants achieved at least a $30 \%$ reduction of misophonia severity based on the Amsterdam Misophonia Scale. The second study added re-evaluating eating norms and stress reduction in a 3-month CBT protocol. The second study reported clinical improvement in $56 \%$ of all completers and $37 \%$ in intention-to-treat participants.

\subsection{Theories of Misophonia}

As noted in a recent misophonia review article, "The lack of a precise theoretical rationale and etiological framework builds a barrier in front of the development of effective management strategies, thereby, posing a challenge for clinicians in the management of misophonic patients in daily practice." (Yilmaz \& Hocaoğlu, 2021).

Misophonia is viewed by many as an emotional response disorder (Brout et al., 2018; Edelstein et al., 2013; Ferrer-Torres, \& Giménez-Llort, 2021; Jager et al., 2020; McKay et al., 2018; Palumbo et al., 2018; Potgieter et al., 2019; Reid et al., 2016; Rouw \& Erfanian, 2018; Schröder et al., 2013; Swedo et al., 2021; Taylor, 2017; Wu et al., 2014) and by others as a physical and emotional reflex disorder (Claiborn et al., 2020; Dozier, 2015a, 2015b, 2015c, 2017; Dozier \& Morrison, 2017; Dozier et al., 2017; Dozier et al., 2020). In one study, 26 participants reported various physical sensations and emotions when exposed to very weak trigger stimuli (Dozier \& Morrison, 2017). Physical responses varied greatly between subjects, with the most prevalent being shoulder and neck flinch. However, each subject's reflex responses were consistent (e.g., participant 1 only had shoulder flinch when hearing eating sounds, and participant 2 had a toe flinch when hearing eating sounds). The researchers proposed that the uniqueness of each participant's physical (muscle) responses supported classical conditioning of a physical reflex as an etiology of misophonia. The initial physical reflex existence was further supported in a study using visual observation of individuals and electromyography to detect the initial physical reflex (Dozier et al., 2020). 
The stimulus-response delay was typically $200 \mathrm{~ms}$ for auditory trigger stimuli and $350 \mathrm{~ms}$ for visual trigger stimuli.

Figure 1 shows the postulated response chain of misophonia as a conditioned aversive reflex disorder (Dozier, 2015c, 2017). Box (1) is the trigger stimulus, whether auditory, visual or another sensory modality. Box (2) indicates the conditioned initial physical reflex, which is directly elicited by the trigger stimulus. This is usually a muscle flinch but can be another physical sensation such as localized pain, sexual sensation, or urge to urinate. Box (3) shows the emotions the individual experiences with a misophonia trigger. These are elicited by the initial physical reflex of the box (2). Box (4) represents the physiological responses that accompany strong emotions. This stress or distress response includes muscle tension, increased heart rate, feelings of warmth, and other physiological responses. Box (5) represents overt behaviors evoked or elicited by misophonia. These include actions to stop, reduce, or escape the trigger stimulus; coping mechanisms such as mimicking; and overt behavior evoked by emotions and stress response including aggression.

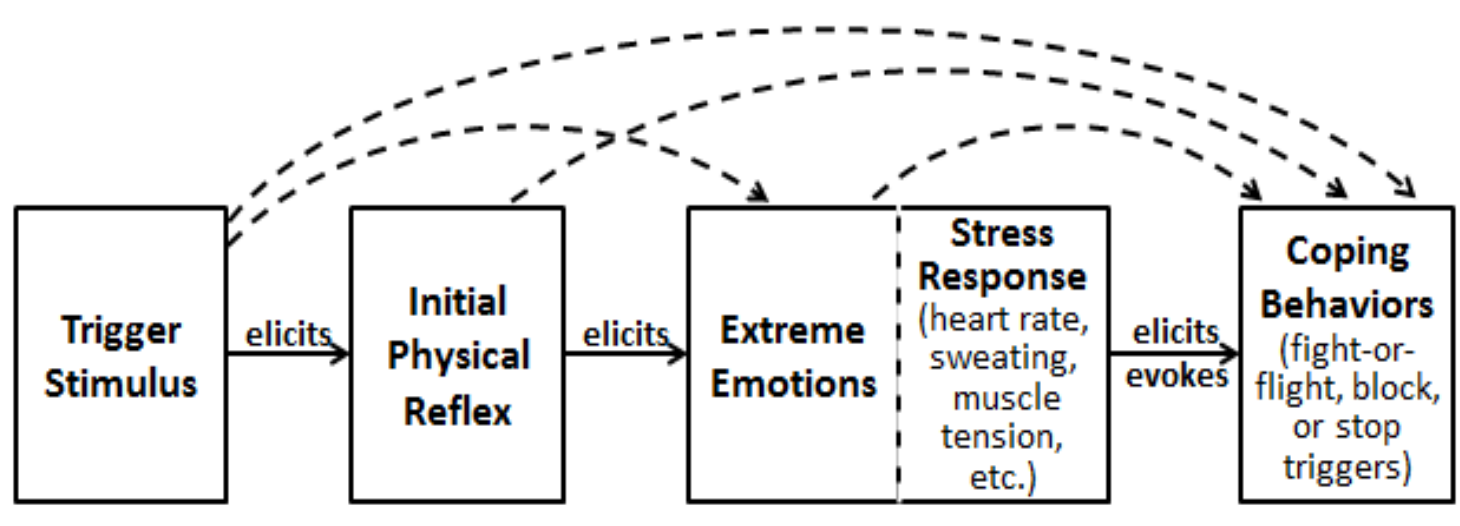

(1)

(2)

(3)

(4)

(5)

Figure 1: Postulated response chain of misophonia as a conditioned aversive reflex disorder showing that the misophonia trigger stimulus (1) elicits a physical (muscle) reflex response (2), which in turn elicits the extreme emotional response (3), stress response (4), and overt behaviors (5).

As support for an initial physical reflex before an emotional response, during behavioral treatment exercises, patients reported situations where the initial physical reflex was experienced without an accompanying emotional response (Dozier, 2015a, 2015b, 2015c). This occurred when exposed to intermittent weak triggers and a continuous positive stimulus (e.g., positive conversation or music).

Dozier (2015b) proposed that misophonia develops as a Pavlovian conditioned muscle reflex through stimulus-response conditioning, as explained by Donahoe and Vegas (2004). This conditioning theory proposes that a muscle response that consistently follows a stimulus becomes a conditioned reflex elicited by that stimulus. Common examples of stimulus-response conditioning include a muscle flinch in response to pagers by surgeons, ringtones by professionals, and chemo-therapy pump sound and vibration by a cancer patient (Dozier, 2017). The conditioned reflex intensifies over time through Pavlovian conditioning because the muscle flinch's sensation causes an immediate tightening of that muscle, thus strengthening the reflex rather than extinguishing it. Dozier theorized the emotional response develops because of the intrusive, aversive nature of the muscle flinch (Dozier, 2015b). Several studies found that aversive stimuli elicit emotional or aggressive responses in humans (Zald et al., 1998; Zald \& Pardo, 1997) and rats (Ulrich \& Azrin, 1962). Functional magnetic resonance imaging (fMRI) studies 
of individuals with misophonia support the theory that the emotions of misophonia are conditioned emotional responses (Kumar et al., 2017; Schröder et al., 2019), given the role of the ventromedial prefrontal cortex in the learning of emotions (Dunsmoor et al., 2019).

As such, misophonia is a disorder comprising Pavlovian conditioned physical reflexes and conditioned emotional responses. Therefore, reducing or eliminating the physical reflex may provide a corresponding reduction in the emotional response.

Because the muscle reflex response is a critical component of misophonia, developing the skill of relaxing muscles on demand is considered beneficial. The ability to relax muscles on demand can be developed using progressive muscle relaxation (PMR; Borkovec \& Sides, 1979; Lehrer et al., 1983) and applied relaxation (progressively relaxing muscles without tensing; Öst, 1987, 1988). Further, PMR has additional benefits of reducing anxiety and depression (Dehghan-Nayeri, \& Adib-Hajbaghery, 2011; Li, et al., 2015; Lolak et al., 2008), and anxiety is commonly reported by people with misophonia (Edelstein et al., 2013; Dozier \& Morrison, 2017; Wu et al., 2014).

\subsection{Authors' backgrounds}

The worldview and experience of an author will influence how data are presented. Therefore, a brief background of the author will be provided. Thomas Dozier is a Master's level board certified behavior analyst. His primary career is a behavioral parenting coach though misophonia now dominates his clinical practice. He was introduced to misophonia by a parent in 2012. Since then he conducted misophonia research and worked personally with misophonic individuals. While working with individuals he listened to their comments and observed some of their immediate physical (muscle) responses, thus leading to the theory that misophonia is a condition where innocuous stimuli elicit both physical (the initial physical reflex) and emotional responses. He is an author of eight journal articles and the book, Understanding and Overcoming Misophonia, A Conditioned Aversive Reflex Disorder. At this time he regularly provides treatment for individuals with misophonia using the treatment protocol described in this case.

\section{Case Presentation}

This case describes routine treatment for misophonia provided by the authors. It was selected for publication because it illustrates the importance of the initial physical reflex of misophonia in this patient, even when the patient reported comorbid bipolar and depression, and extreme social isolation and avoidance due to her misophonia.

As an ethical consideration, this treatment was designed to not require the patient to experience severe trigger stimuli as a component of treatment. Rather, it was designed to allow the patient to respond to misophonic triggers in a way that would reduce their immediate and future response severity. The proposed exposure to weak trigger stimuli was previously reported without adverse effect (Dozier, 2015a, 2015c).

Olivia (pseudonym) was a 47-year-old White single woman. She held a Ph.D. degree and was employed for 15 years in genetics and immunology research. She worked her last 5 years as a scientific advisor for a biological research company. Unfortunately, she quit her job because "misophonia made it impossible for [her] to work in a public space." Despite asking for accommodations, her boss did not believe in misophonia as an actual disorder. Olivia quit her job suddenly when her boss intentionally 
clicked his pen several times in her face after she reported pen clicking as a misophonia trigger. Without income, Olivia was forced to move in with her parents at age 45 . Olivia stated she avoided most social relationships and completely avoided romantic relationships because of her misophonia. She was diagnosed with Bipolar II, and she self-reported "mild OCD tendencies" and worsening social anxiety. She reported spending about $95 \%$ of her time alone or wearing headphones to block misophonic trigger stimuli. She reported developing misophonia at age 22, and her misophonia worsened over the years. She presented as well-groomed, articulate, and highly motivated for treatment.

\subsection{Presenting Complaints}

Olivia contacted the author by email seeking treatment that would include an intensive therapy protocol where she could work with professionals to stop the uncontrollable rage felt from sound triggers. She stated her misophonia was so intolerable that she was not able to function normally. Because of misophonia, she was afraid to seek out a job, only left her house about twice a month, and had multiple breakdowns in public due to misophonic triggers. Olivia stated her distress from misophonia induced suicidal thoughts because she was "all out of coping skills." She self-diagnosed her condition as misophonia, based upon internet research. She also wanted help with the secondary conditions of depression and anxiety from not participating in public life. She reported that her misophonia was so severe that she had no enjoyment in life, was afraid to be around other people, and felt continual hopelessness and despair, eliminating virtually all joy in life.

\subsection{History}

Olivia reported that her misophonia began about age 22 to the sound of a car turn signal. Up to that time, she was a competitive athlete. She "never rested" as an athlete or university student. After she was in a car accident, she went on an international rock-climbing trip with a fractured sternum and was in a great deal of pain. During this time, she first experienced misophonia. After this, she had sinus repair surgery and never fully recovered. She went from being a competitive athlete to being bedridden because of chronic fatigue. She had severe depression during her Master's and Ph.D. programs, and after she completed her Ph.D., she was so exhausted, she recuperated for 3 months. She reported that her misophonia became severe around age 30. Olivia had a diagnosis of Bipolar II and took the moodstabilizer Lamotrigine. She later reported a diagnosis of Complex PTSD from chronic stress and insomnia during her Ph.D. program. She took Ambien every night for sleep.

\subsection{Assessment and Data Analysis}

A battery of assessment forms was emailed to the client, which she completed and returned by email. Olivia described her response to trigger stimuli as intolerable. Results from the assessments indicated that triggers almost always elicited feelings of anger, rage, hopelessness, guilt, anxiety, sadness, fear of more triggers, and a desire to escape or flee. Additionally, she often fantasized about physically lashing out at the person making the trigger. She rated her physiological response as "physical sensation which can be best described as emotional pain with which she could not cope." She was unaware of any specific muscle responses elicited by trigger stimuli which might be her initial physical reflex. She identified the auditory triggers as clock ticking, pens clicking, eating/drinking mouth sounds, footsteps through the ceiling, loud breathing, gum popping, someone scratching skin, high pitched voices, female vocal fry, stylized female singers, and children whining, crying, or screaming. Her visual triggers were open mouth chewing and picking at fingernails. The severity questionnaires included the Misophonia 
Assessment Questionnaire (MAQ; Dozier, 2015a), Misophonia Impact Survey (MIS; (Dozier, 2017), and Amsterdam Misophonia Scale (A-MISO-S; Schröder et al., 2013).

The MAQ consists of 21 questions about the negative impact of misophonia on the person's activities, thoughts, and feelings (see Table 1). Each question is rated 0 to 3 , with 0 being "not at all" and 3 being "almost all of the time," yielding a maximum possible sum score of 63 . The Misophonia Impact Survey rates how misophonia interferes in five domains of participation in life on a 0-10 scale or "not applicable" for each domain (see Table 2). These areas (family, romantic relationships, social/leisure with others, work/school, and individual activities and alone time) were selected to indicate the impact of misophonia in common environments of daily life that are applicable to most individuals. Because of "not applicable" categories of social/leisure with others and work/school, Olivia's MIS range was 0-30. The AMISO-S survey is a derivation of the Y-BOCS and rates 6 factors of misophonia - time, interference, distress, resistance to thoughts, control over thoughts, and avoidance (0-24 range). Both the MAQ and A-MISO-S were modified to comprehend that misophonia commonly includes auditory and visual triggers (e.g., change "sound" to "trigger"). These three instruments are not validated. The severity assessments were based solely on the self-report of the client. Data were analyzed by calculating the recovery percentage of each measure, using the formula recovery-percentage equals (initial-score - present score) / initial-score.

Table 1. Misophonia Assessment Questionnaire. The sum of all scores of this assessment is an indicator of the severity of misophonia.

\begin{tabular}{|l|l|l|l|l|}
\hline $\begin{array}{l}\text { RATING SCALE: } \\
0=\text { not at all, } 1=\text { some of the time, } 2 \text { = a good deal of the time, } 3=\text { almost all } \\
\text { the time }\end{array}$ & & & \\
\hline 1. Misophonia currently makes me unhappy & 0 & 1 & 2 & 3 \\
\hline 2. Misophonia currently creates problems for me. & 0 & 1 & 2 & 3 \\
\hline 3. Misophonia has recently made me feel angry. & 0 & 1 & 2 & 3 \\
\hline 4. I feel that no one understands my problems caused by misophonia. & 0 & 1 & 2 & 3 \\
\hline 5. My response to certain triggers does not seem to have a known cause. & 0 & 1 & 2 & 3 \\
\hline 6. My response to certain triggers currently makes me feel helpless. & 0 & 1 & 2 & 3 \\
\hline 7. My responses to triggers currently interfere with my social life. & 0 & 1 & 2 & 3 \\
\hline 8. Misophonia currently makes me feel isolated. & 0 & 1 & 2 & 3 \\
\hline 9. Misophonia has recently created problems for me in groups. & 0 & 1 & 2 & 3 \\
\hline 10. Misophonia negatively affects my work or school life. & 0 & 1 & 2 & 3 \\
\hline 11. My issues due to misophonia currently make me feel frustrated. & 0 & 1 & 2 & 3 \\
\hline 12. Misophonia currently impacts my entire life negatively. & 0 & 1 & 2 & 3 \\
\hline 13. Misophonia has recently caused me to feel guilty. & 0 & 1 & 2 & 3 \\
\hline 14. My experience of misophonia is classified as 'crazy.' & 0 & 1 & 2 & 3 \\
\hline 15. I feel that no one can help me with my misophonia. & 0 & 1 & 2 & 3 \\
\hline 16. Misophonia currently makes me feel hopeless. & 0 & 1 & 2 & 3 \\
\hline 17. I feel that my misophonia will only get worse with time. & 0 & 1 & 2 & 3 \\
\hline 18. Misophonia currently impacts my family relationships. & 0 & 1 & 2 & 3 \\
\hline 19. Misophonia has recently affected my ability to be with other people. & 0 & 1 & 2 & 3 \\
\hline 20. My experience of misophonia has not been recognized as legitimate. & 0 & 1 & 2 & 3 \\
\hline 21. I am worried that my whole life will be affected by misophonia. & 0 & 1 & 2 & 3 \\
\hline
\end{tabular}


Table 2. Misophonia Impact Survey. This survey tracks impairment from misophonia in five domains of participation in life as follows: family, romantic relationships, social/leisure with others, work/ school, and individual activities and alone time. The sum of all scores of this assessment is an indicator of the severity of misophonia.

Questions of Misophonia Impact Survey

1. Rate how misophonia has interfered with family life in the past 2 weeks*. (If you have avoided these activities because of misophonia, include that factor in your rating.)

2. Rate how misophonia has interfered with romantic relationships in the past 2 weeks*. (If you

3. Rate how misophonia has interfered with your social life and leisure activities with others in the past 2 weeks*. (If you avoid these activities because of misophonia, include that factor in your rating.)

4. Rate how misophonia has interfered with your work/school work, including unpaid volunteer work, training, or similar activities in the past 2 weeks*. (If you avoid these activities because of misophonia, include that factor in your rating.)

5. Rate how misophonia has interfered with your individual activities and alone time in the past 2 weeks*. (If you avoid certain activities because of misophonia include that factor in your rating.)

Rating Scale for Each Question

None Mildly Moderately Severely Extremely Not-applicable

$\begin{array}{lllllllllllll}0 & 1 & 2 & 3 & 4 & 5 & 6 & 7 & 8 & 9 & 10 & \text { N/A }\end{array}$

* The initial assessment used a time of 2-weeks. Assessment during treatment used a time of 1-week.

\subsection{Case Conceptualization}

As mentioned previously, the misophonic response is conceptualized as a Pavlovian conditioned physical reflex followed by conditioned emotional responses, as shown in Figure 1 . The goal of treatment was to reduce the initial physical reflex, thereby reducing the subsequent emotional response. The assessment indicated Olivia's initial physical reflex was a distinct muscle response, so counterconditioning was expected to reduce or eliminate that reflex. Stimulus-response Pavlovian conditioning theory purports that the brain develops and maintains a stimulus's muscle reflex response because it occurs after a stimulus. It is proposed that the initial physical reflex of misophonia persists because the emotional responses (e.g., anger, disgust, anxiety) have accompanying physical responses that cause the initial physical reflex muscles to tense in the first few seconds after a trigger, thus strengthening the conditioned reflex Dozier, 2015b). If the individual can relax the initial physical reflex muscle before a trigger and continue relaxing that muscle through and after the trigger, it is reasonable to presume that the reflex would be weakened. Indeed, this was the premise in an earlier case where the patient engaged in daily progressive muscle relaxation training and relaxed her muscles while listening to music and hearing intermittent weak triggers using the Misophonia Trigger Tamer app (Dozier, 2015c).

Repeated progressive muscle relaxation training (PMR) develops the behavioral skill of relaxing muscles on demand. Additionally, PMR can help reduce anxiety, depression, and stress, and improve overall health and well-being, which typically reduce the severity of misophonia. Treatment included three sessions ( 1 hour total) of progressive muscle relaxation per day initially, with additional applied relaxation exercises after completing 14 to 20 PMR sessions.

Counterconditioning the initial physical reflex can be accomplished using planned activities that pair a positive activity with a weak misophonia trigger (Dozier, 2015a, 2015c). For counterconditioning with 
in vivo triggers, the individual may use noise as needed (ambient or headphones) to reduce the severity of the trigger so he/she can relax the initial physical reflex muscles before and through the trigger event.

Misophonia often becomes more severe through typical exposure to trigger stimuli - the misophonic response is strengthened and new trigger stimuli develop. Adding background noise to the environment is a standard method to reduce the severity of auditory triggers (Dozier, 2017). Selective avoidance is a common practice to minimize the incidence of distress from misophonic triggers. The use of background noise and selective avoidance was an initial component of treatment.

In summary, the treatment included 1) education on misophonia reflexes, 2) relaxation training using progressive muscle relaxation and applied relaxation, 3) counterconditioning, 4) avoiding misophonia distress (particularly prolonged distress), and 5) ongoing guidance and support from the therapist. This is relaxation and counterconditioning therapy (RCT) for misophonia.

\section{Treatment and Assessment of Progress}

The proposed treatment was explained in the initial email message and the first session as a behavioral treatment, including muscle relaxation training and counterconditioning of the misophonia reflexes. The treatment plan included weekly sessions for the first 3 weeks and then sessions at longer intervals as the response to treatment warranted. The initial assessment/therapy session included education on the reflexes of misophonia as shown in Figure 1 and helping Olivia determine her initial physical reflex to trigger stimuli. The reflex test procedure consists of making very weak trigger stimuli and having the client report any physical sensations (Dozier \& Morrison, 2017). The use of very weak trigger stimuli is required to avoid the strong misophonic response where extreme emotions and physiological distress dwarf the initial physical reflex sensation. The initial physical reflex test was conducted for each trigger class because different trigger classes (e.g., chewing vs. pen clicking) can elicit different initial physical reflexes. In one study , $42 \%$ of participants reported different physical responses for different classes of triggers (Dozier \& Morrison, 2017).

Olivia was aware that she tensed her jaw and fists when exposed to triggers but did not understand this to be an immediate reflex elicited by the trigger. With testing, she reported that pen clicking, eating, nail picking, and nose whistle all elicited a jaw-clench reflex. Pen clicking elicited a jaw-clinch and contraction in the back of her neck. Picking nails elicited a jaw-clinch and a sensation behind her ears. Clinching her fists was, therefore, a secondary response and not part of her initial physical reflex. Olivia was assigned the task of testing other classes of triggers on her own, which included the sounds of flip flops, clocks ticking, children crying, women vocal fry, and women stylized singing.

Using misophoniatreatment.com/pmr as a resource, Olivia was taught about progressive muscle relaxation (PMR). She was assigned homework to watch the training video on that page and complete one hour of PMR per day, consisting of one long session (25 minutes) and two short sessions (15 minutes each) included on that website. She was instructed that if anything in the PMR audio triggered her to stop immediately, discontinue use of that file, and report the problem to her therapist. Because she had trouble sleeping, she was also asked to do an additional PMR session in bed while she was trying to fall asleep.

Olivia was instructed to try to relax and focus her attention away from triggers. Specifically, she was asked to relax her muscles when going into a possible trigger situation, relax immediately after being 
triggered, and relax and stay relaxed whenever there was a continuing trigger situation, focusing on relaxing her jaw. The importance of relaxing her jaw before and through triggers was emphasized as the counterconditioning mechanism that reduces future responses to triggers. This was emphasized in this session and all of the follow-on sessions. However, Olivia was advised to avoid prolonged misophonia distress, because this could increase her misophonia severity and be counterproductive to her treatment. She was specifically advised to avoid staying in a situation where she was exposed to triggers and her level of distress was increasing. If she experienced triggers and her level of distress was declining or steady at a moderate level, then she could remain in that environment if she chose to do so.

The second session occurred 7 days later. Olivia reported that the short PMR audio triggered her, but she was doing the long PMR each morning and at bedtime, with the positive benefit of going to sleep around midnight rather than 3:00 am. Olivia reported that her misophonia was worse the first 3 days after the first session because she was hyper-aware of her jaw reflex. The third day after the first session, she had a positive experience of the benefit of relaxing. Olivia and her mother decided to pull weeds from an elderly neighbor's flower garden. While they worked, school let out, and children started playing outside and shrieking. Instantly, Olivia felt distressed, which she rated as 9 of 10 . She wanted to continue doing the service activity, so she tried to relax. Almost immediately, she felt her level of distress slightly decreasing. After 5 minutes, she rated her distress as 5 of 10, then 2 of 10 after 15 minutes. After 20 minutes, she said she was unaware of the children screaming.

Even with this positive experience, Olivia did not go out that week because of her fear of triggers, and she did not conduct any tests to determine the initial physical reflex of her other triggers. The principle of spontaneous recovery of conditioned reflexes was explained so she would understand that even though the "children yelling" trigger had extinguished once, she would likely experience a significant trigger response the next time she heard that sound. However, her response would be less than the previous experience and extinguish quicker. She was introduced to the Misophonia Trigger Tamer app as a tool to experience misophonia triggers to countercondition the initial physical reflex gently. She was taught two additional exercises - sequential relaxation (i.e., applied relaxation) and total instant relaxation (ragdoll), relaxing all muscles as quickly as possible. She was asked to do the sequential relaxation exercise three times a day and ragdoll five times a day in a trigger-free environment. Then, she was to "ragdoll" when going into a possible trigger situation, immediately after a trigger, and before and through triggers. She was reminded that only the last of these would decrease her future misophonic response. Olivia completed the Misophonia Assessment Questionnaire (MAQ), giving oral responses as the therapist read the questions. Her MAQ score was 37 vs. 63 at intake. No data was taken on the Misophonia Impact Survey (MIS). In all future sessions, Olivia orally gave her responses to the MAQ and MIS at the end of each session (see Table 3).

Table 3. Misophonia Severity Ratings and Response to Treatment

\begin{tabular}{|c|c|c|c|c|c|c|c|c|c|c|}
\hline \multirow{2}{*}{ Session } & \multirow{2}{*}{ Weeks } & \multicolumn{4}{|c|}{ MAQ } & \multicolumn{4}{c|}{ MIS } & \multicolumn{3}{c|}{ A-MISO-S } \\
\cline { 3 - 12 } & & $\begin{array}{c}\text { Number } \\
(0-63)\end{array}$ & $\begin{array}{c}\text { Percent } \\
\text { of Max }\end{array}$ & $\begin{array}{c}\text { Recovery } \\
\text { Percent }\end{array}$ & $\begin{array}{c}\text { Number } \\
(0-30)\end{array}$ & $\begin{array}{c}\text { Percent } \\
\text { of Max }\end{array}$ & $\begin{array}{c}\text { Recovery } \\
\text { Percent }\end{array}$ & $\begin{array}{c}\text { Number } \\
(0-24)\end{array}$ & $\begin{array}{c}\text { Percent } \\
\text { of Max }\end{array}$ & $\begin{array}{c}\text { Recovery } \\
\text { Percent }\end{array}$ \\
\hline 1 & 0 & 63 & $100 \%$ & $0 \%$ & 26 & $80 \%$ & $0 \%$ & 21 & $88 \%$ & $0 \%$ \\
\hline 2 & 1 & 37 & $58.7 \%$ & $41.3 \%$ & - & - & - & - & - & - \\
\hline 3 & 4 & 22 & $34.9 \%$ & $65.1 \%$ & 9 & $30 \%$ & $65.4 \%$ & - & - & - \\
\hline 4 & 7 & 17 & $27.0 \%$ & $73.0 \%$ & 6 & $20 \%$ & $76.9 \%$ & - & - & - \\
\hline 5 & 10 & 3 & $4.8 \%$ & $95.2 \%$ & 1 & $3 \%$ & $96.2 \%$ & - & - & - \\
\hline
\end{tabular}




\begin{tabular}{|c|c|c|c|c|c|c|c|c|c|c|}
$\begin{array}{c}\text { End of } \\
\text { Treatment }\end{array}$ & 13 & 5 & $7.9 \%$ & $92.1 \%$ & 2 & $7 \%$ & $92.3 \%$ & 8 & $33.3 \%$ & $61.9 \%$ \\
\hline Follow-up & 50 & 4 & $6.3 \%$ & $93.7 \%$ & 0 & $0 \%$ & $100.0 \%$ & 3 & $12.5 \%$ & $85.7 \%$ \\
\hline
\end{tabular}

The third session occurred three weeks after the second because of the death of a relative. She reported that she had not done any PMR sessions or used the Misophonia Trigger Tamer app, but her misophonia was markedly reduced. She took an airplane flight to visit family and was in public many times, which went very well. She was pleased that she effectively managed a 5-hour wait in a "packed" airport. She credited her use of new management tools which were to take quick action to escape the trigger if needed, relax into situations, and relax and breathe slowly when there were triggers. She felt she was making real progress with her misophonia and anxiety. To help her anxiety, she set an hourly timer on her phone and relaxed when alerted, noticing that her jaw was tense about $90 \%$ of the time. For counterconditioning experiences, she ate one meal a day with her mother and spent time with her father while he ate popcorn or bananas. During both of these, she engaged in positive conversation and worked to keep her jaw relaxed. She reported that her initial physical reflex was her jaw clinching for all the triggers not tested in the assessment, as far as she could tell. Because of her excellent progress and the planned treatment activities, it was agreed the next session would be three weeks later.

In the fourth session, she reported that she was very agitated for the last 2 weeks. She had done PMR with audio for the first 2 weeks but had only done self-guided PMR at bedtime for the past week. She reported a positive experience going to the movie theater. She relaxed when noisy people triggered her and felt anger for the first one to two minutes, but by 15 to 20 minutes, she was calm and enjoyed the movie. She had also reduced her Ambien at bedtime from $10 \mathrm{mg}$ to $5 \mathrm{mg}$ and slept well. The treatment recommended for misophonia was to resume PMR twice a day with the audio file, use an interval timer app as a cue to relax every 30 minutes, and add ambient noise in situations where that would help. She was also asked to practice mindfulness by taking a walk or doing an activity for 10 to 20 minutes, paying constant attention to what she was doing and her surroundings at that moment, and to be mindful of things in her environment when she was triggered as a way to focus her attention away from the trigger. Because she was responding well to the treatment, the next session was scheduled for three weeks later.

In the fifth session, Olivia reported that her mental state was excellent. She was happy and looking forward to things. She completed one PMR session every night with audio and 2 to 3 times a week in the morning. She reported that she was very anxious and worried about triggers before the last session, but that had significantly decreased. She had used the interval timer app to alert herself every half hour and relaxed her muscles with extra focus on her jaw. She had a great trip to Las Vegas to visit her brother. He was respectful of her misophonia and helped her manage it. When needed, she wore earplugs or listened to rock music, and she managed very well. She was not stressed and constantly thinking about how she could escape in case there were triggers. Overall, for the past 3 weeks, she was more relaxed and less avoidant. She felt she still tended to revert to her old misophonia response when triggered, but she would relax and continue with the activity. She even had a 3-hour conversation with her father while he was eating, and she was not "bothered." Her assignment for homework was to continue the same PMR schedule and relax through trigger situations. The next session was scheduled for 5 weeks later.

After 3 weeks, Olivia decided that she did not need to meet again, so she cancelled the upcoming appointment, and a final severity rating was collected by telephone. Her overall response to treatment and follow-up is shown in Figure 2. 


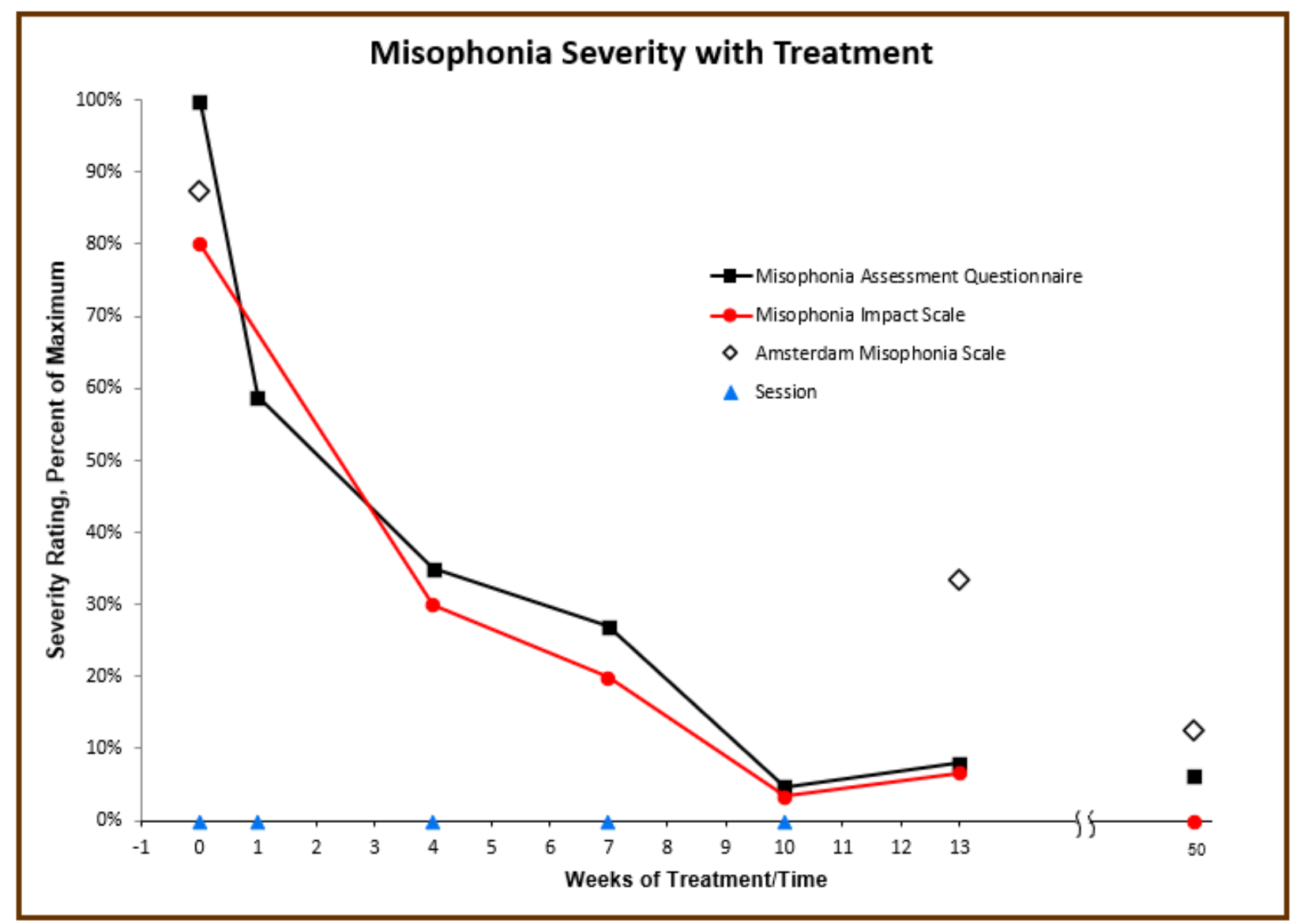

Figure 2. Misophonia severity measures vs. weeks of treatment. Severity ratings are shown as percent of maximum for each measure.

\subsection{Data Analysis}

Recovery percentage for each measure is shown in Table 3. Recovery percentages for the Misophonia Assessment Questionnaire, Misophonia Impact Survey, and Amsterdam Misophonia Scale were $92.1 \%, 92.3 \%$, and $61.9 \%$ respectively at end of treatment, and declined further at follow-up to $93.7 \%, 100 \%$, and $85.7 \%$. Of particular note is the essentially monotonic decline in misophonia severity during the 13-week treatment.

\subsection{Complicating Factors}

Because of Olivia's extreme avoidance of trigger stimuli, she was resistant to counterconditioning of misophonia triggers. Conceptually it made sense, but based on her experience with triggers, it was frightening. Fortunately, she had a very positive experience with counterconditioning of a real-life trigger a few days after the first session in a situation where there was natural motivation to endure the triggers. This success enabled her to be more willing to engage in counterconditioning activities that required experiencing weak triggers. Because she had a distinct initial physical reflex that was a muscle used extensively in tension and relaxation in ordinary life, she was able to relax her jaw when triggered, and she experienced a quick reduction in the severity of her misophonic response. Had this not occurred, 
treatment would likely have taken longer, and counterconditioning exercises would have been introduced more gradually using recorded triggers.

\subsection{Access and Barriers to Care}

Access and barriers to care will be covered in logistical and intrapsychic issues. Treatment was conducted via VSee, a program designed for telehealth, so confidentiality was maintained, and location and transportation did not pose a problem. The homework requirements of an hour per day did not place a significant burden on the patient but required a commitment on her part to accomplish this treatment.

A general barrier of counterconditioning treatment is the extreme aversive response of individuals to misophonia triggers and general avoidance of triggers (Dozier et al., 2017; Schröder et al., 2013), thus decreasing adherence and motivation. Another barrier is adherence to progressive muscle relaxation exercises (Hoelscher et al., 1984). The therapist engaged Olivia with motivational interviewing conversations to increase adherence, emphasizing the benefit of adherence to protocols. Olivia was adherent with relaxation training but very resistant to counterconditioning because it required exposure to trigger stimuli. Even though the therapist explained it would include very weak stimuli, such as was used in determining her initial physical reflex, she did not do the counterconditioning with the Trigger Tamer app. Because Olivia was exposed to trigger stimuli through regular life events in the first week of treatment and was successful with relaxation/counterconditioning techniques, she was willing to plan counterconditioning exercises to facilitate her treatment progress. Had this unintended exposer not occurred, a telehealth limitation would have been increased difficulty in starting counterconditioning activities.

\subsection{Follow-Up}

One follow-up session was conducted about a year after the start of treatment. Olivia reported that she was more social than she was in years and didn't say no to doing things. She reported not using headphones or earplugs to avoid sounds, except for eating with her father and sleeping. Her father's loud chewing was her only remaining consistent misophonia trigger, and the severity was significantly reduced. She only experienced significant triggers once or twice a month, and then only when she was tired from insomnia due to hot flashes. She still noticed most old trigger sounds, but mild triggers were no longer noticed. She was considering going back to work but wanted to get a job where there would not be danger of being "trapped" with misophonia triggers. After having misophonia for 25 years, she expressed a need to develop a new identity and felt she needed additional counseling for this. Olivia had a very positive response to treatment, but several psychological issues remained due to her many years of misophonia distress and comorbid conditions.

\section{Discussion}

This case supports the theory that misophonia is a condition where subtle stimuli elicit physical and emotional reflex responses. More specifically, it supports the theory shown in Figure 1 that misophonia generally includes an initial physical reflex elicited by trigger stimuli. In this case, the patient had an initial physical reflex (jaw clinch) and when that when that reflex was counterconditioned by relaxing that muscle in the presence of misophonic triggers, her misophonia severity decreased sharply. This etiology theory of misophonia purports that the physical reflex develops through stimulus-response classical conditioning, and in some cases, it may be counterconditioned and extinguished by relaxing the muscles 
of the initial physical reflex before, through, and immediately following the misophonic trigger. This case also illustrates the benefit of intensive progressive muscle relaxation and applied relaxation for both the ability to relax muscles on-demand and improvement in overall health and well-being. Both of these were likely factors in the reduction of Olivia's misophonia severity. This case was a behavioral intervention for misophonia and did not address psychological factors. There was no cognitive restructuring or mental evaluation of Olivia's misophonic experience in this treatment - a treatment that significantly reduced her misophonia severity including her emotional responses. Psychotherapy may have enhanced Olivia's recovery as she expressed a desire to have therapy for issues that remained after her misophonic responses were virtually eliminated.

It is important to note that some misophonia sufferers do not experience a skeletal muscle response as the initial physical reflex. In one study, $23 \%$ of participants reported an immediate sensation of nausea, warmth, numbness, or sexual sensation (Dozier\& Morrison, 2017). These responses would likely not respond to the counterconditioning exercises of relaxing muscles in trigger situations; however, it is uncertain whether these reflexes could be counterconditioned when paired with very positive stimuli. The counterconditioning stimuli are unique to each patient and could include listening to music, dancing, outdoor activity, or even looking at pictures that fill their soul with joy. In one case, the counterconditioning stimuli included talking about very positive life accomplishments (Dozier, 2015a). Counterconditioning could include both very positive stimuli and relaxing muscles as described by Dozier (2015c). An outline of relaxation and counterconditioning therapy (RCT) for misophonia is shown in Table 4.

Table 4. Components of Relaxation and Counterconditioning Therapy (RCT) for misophonia.

\begin{tabular}{|l|l|}
\hline Component & Activity \\
\hline 1 & Education on misophonia reflexes (see Figure 1) and counterconditioning \\
\hline 2 & Intensive relaxation training using progressive muscle relaxation and applied relaxation \\
\hline 3 & Counterconditioning the initial physical reflex \\
\hline 4 & Avoiding misophonia distress (particularly prolonged distress) \\
\hline 5 & Ongoing guidance and support \\
\hline
\end{tabular}

\subsection{Research Implications}

Misophonia is being researched as a condition in which the disorder stems from hyper focus on specific sounds and immediate negative affective reaction, which lead to avoidance, isolation, and functional impairment. The treatment studies described in the introduction and those in progress are attempting to provide treatment of symptoms without "a precise theoretical rationale and etiological framework" (Yilmaz \& Hocaoğlu, 2021). No etiology theories have been investigated, including the one shown in Figure 1, which was proposed in 2015 (Dozier, 2015b). Psychological literature is virtually devoid of research of common Pavlovian reflexes such as a physical response of a doctor to their pager, of a professional to specific phone ring tones, or of a cancer patient to their chemo pump (Dozier, 2017). In an unpublished pilot survey, 6 of 10 physicians reported having an immediate physical response to their pager (Dozier, 2017). Pavlovian reflexes may be ubiquitous, yet there is no research on this topic. Further research on the composition and etiology of misophonia is desperately needed. 


\subsection{Further Treatment Implications}

This treatment protocol was sufficient for Olivia, but as a general treatment protocol, it may be enhanced by including other cognitive behavioral therapy techniques as appropriate. These might include cognitive restructuring of thought associated with triggers, mindful shifting of attention after being triggered, and treatment for comorbid disorders.

General awareness of misophonia among therapists and helping professionals of all disciplines is needed to address the unique needs of misophonia sufferers, and effective treatment protocols need to be developed and empirically validated. This seems important considering the high prevalence of misophonia in the general population and higher prevalence in clinical populations as described in the introduction.

\subsection{Limitations}

Limitations of this case study include self-report measures, diagnostic concerns, comorbidity, lack of experimental control, and generalizability. All measures of misophonia severity were self-report of the patient and therefore have a limitation of reliability of data. Even the assessment of the patient's initial physical reflex conducted by the therapist relied on self-report of the patient after experiencing weak triggers. Misophonia is not included in the DSM-5 or ICD-11 and symptomology of misophonia overlaps with other disorders (Taylor, 2017). This may affect the reliability of the self-report severity. For example, the patient reported comorbid insomnia, which significantly improved during this treatment. The effect of this on her misophonia severity cannot be quantified. Similar issues are related to her report of PTSD and depression, which were not assessed during this treatment and may have affected the patients misophonia severity ratings. This case is a simple A-B intervention, so there is no demonstration that the favorable response was actually due to the treatment. Although misophonia severity steadily declined during treatment and the patient did not report any notable events, there could have been other factors that caused the reported decline in misophonia severity. Finally, as a case study, generalizability of this study is limited because many variables, including the variation in the initial physical response of individuals with misophonia.

\section{Conclusions}

This single-case study illustrates the misophonia theory shown in Figure 1 and is repeated here in Figure 3 with highlighted, that through the initial physical reflex the elicitation of the extreme emotional response. This theory purports that misophonia is a conditioned aversive reflex disorder (CARD) which may be a more appropriate name than misophonia. It begins when a Pavlovian physical reflex (the initial physical reflex) develops to a subtle, innocuous stimulus. The physical and emotional reflexes are then strengthened through typical exposure to trigger stimuli. But when the initial physical reflex's muscle is held relaxed during exposure to trigger stimuli, the initial physical reflex and the emotional reflex can diminish and extinguish. This theory should be an essential consideration for research of the etiology, expansion, maintenance, and treatment of CARD (misophonia). 


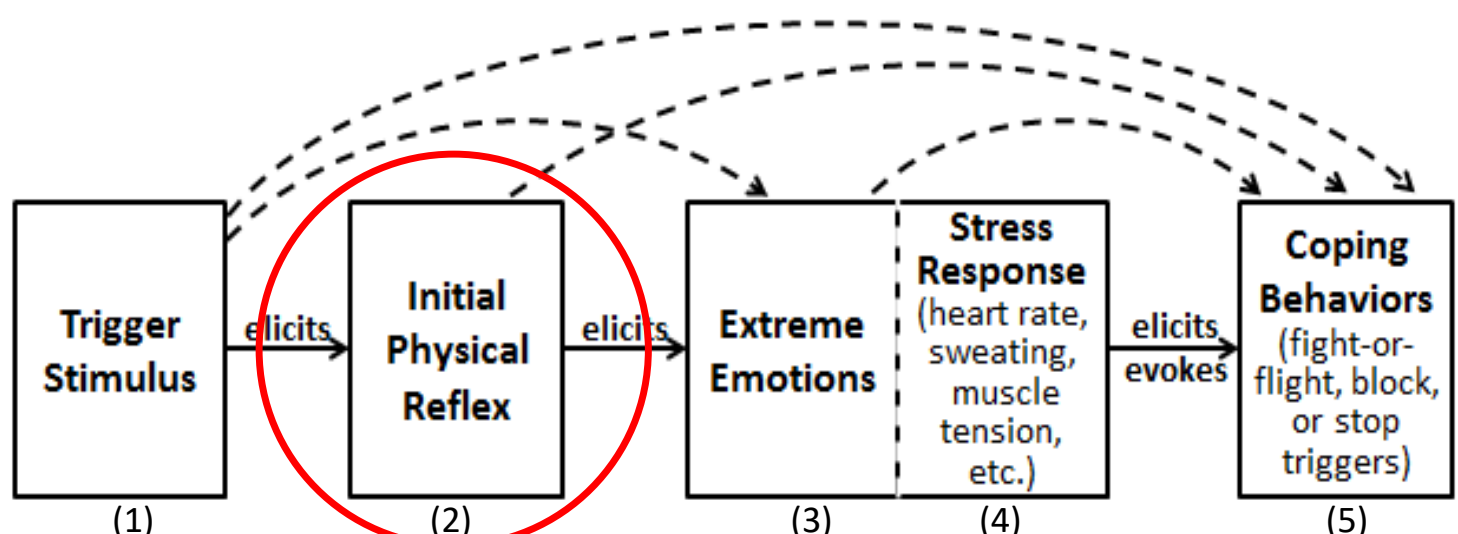

Figure 3. Postulated misophonia response chain as a conditioned aversive reflex disorder, showing that the misophonia trigger stimulus (1) elicits a physical (muscle) reflex response (2), which in turn elicits the extreme emotional response (3). The distress of the emotions that trigger the stress response (4) and the coping (overt) behaviors (5) are then elicited and evoked.

\section{References}

Bernstein, R. E., Angell, K. L., \& Dehle, C. M. (2013). A brief course of cognitive behavioural therapy for the treatment of misophonia: A case example. The Cognitive Behaviour Therapist, 6(10), 1-13. https://doi.org/10.1017/S1754470X13000172

Borkovec, T. D., \& Sides, J. K. (1979). Critical procedural variables related to the physiological effects of progressive relaxation: A review. Behaviour Research and Therapy, 17(2), 119-125. doi:10.1016/0005-7967(79)90020-2

Brout, J. J., Edelstein, M., Erfanian, M., Mannino, M., Miller, L. J., Rouw, R., ... \& Rosenthal, M. Z. (2018). Investigating misophonia: A review of the empirical literature, clinical implications, and a research agenda. Frontiers in Neuroscience, 12, 36.

Cash, T. V. (2015). Decreased sound tolerance (DST): Prevalence, clinical correlates, and development of a DST assessment instrument.

Claiborn, J. M., Dozier, T. H., Hart, S. L., \& Lee, J. (2020). Self-Identified Misophonia Phenomenology, Impact, and Clinical Correlates. Psychological Thought, 13(2).

Dehghan-Nayeri, N., \& Adib-Hajbaghery, M. (2011). Effects of progressive relaxation on anxiety and quality of life in female students: A non-randomized controlled trial. Complementary Therapies in Medicine, 19(4), 194-200. doi: 10.1016/j.ctim.2011.06.002

Donahoe, J. W., \& Vegas, R. (2004). Pavlovian Conditioning: The CS-UR Relation. Journal of Experimental Psychology: Animal Behavior Processes, 30(1), 17-33. doi:10.1037/00977403.30.1.17

Dozier, T. H. (2015a). Counter Conditioning treatment for misophonia. Clinical Case Studies, 14, 1-14. Doi: $10.1177 / 1534650114566924$

Dozier, T. H. (2015b). Etiology, composition, development, and maintenance of misophonia: A conditioned aversive reflex disorder. Psychological Thought, 8, 1-16. doi: 10.5964/psyct.v8i1.132

Dozier, T. H. (2015c). Treating the initial physical reflex of misophonia with the neural repatterning technique: A counterconditioning procedure. Psychological Thought, 8(2), 189-210. doi:10.5964/psyct.v8i2.138

Dozier, T. H. (2017). Understanding and overcoming misophonia: A conditioned aversive reflex disorder. 2nd ed. Livermore (CA): Misophonia Treatment Institute.

Dozier, T. H., Grampp, L., \& Lopez, M. (2020). Misophonia: Evidence for an elicited initial physical response. Universal Journal of Psychology 8(2): 27-35. doi: 10.13189/ujp.2020.080202 
Dozier, T. H., Lopez, M., \& Pearson, C. (2017). Proposed diagnostic criteria for misophonia: A multisensory conditioned aversive reflex disorder. Frontiers in psychology, 8, 1975.

Dozier, T. H., \& Morrison, K. L. (2017). Phenomenology of misophonia: initial physical and emotional responses. American Journal of Psychology, 130(4), 431-438. doi: 10.5406/amerjpsyc.130.4.0431

Dunsmoor, J. E., Kroes, M. C., Li, J., Daw, N. D., Simpson, H. B., \& Phelps, E. A. (2019). Role of human ventromedial prefrontal cortex in learning and recall of enhanced extinction. Journal of Neuroscience, 39(17), 3264-3276.

Edelstein, M., Brang, D., Rouw, R., \& Ramachandran, V.S. (2013). Misophonia: Physiological investigations and case descriptions. Frontiers in Human Neuroscience, 7 (URL=http://www.frontiersin.org/Journal/Abstract.aspx?s=537\&name=human_neuroscience \&AR T_DOI=10.3389/fnhum.2013.00296)

Ferrer-Torres, A., \& Giménez-Llort, L. (2021). Confinement and the hatred of sound in times of COVID19: a Molotov cocktail for people with misophonia. Frontiers in Psychiatry, 12, 663.4.j

Hoelscher, T. J., Lichstein, K. L., \& Rosenthal, T. L. (1984). Objective vs subjective assessment of relaxation compliance among anxious individuals. Behaviour research and therapy, 22(2), 187193.

Jager, I., de Koning, P., Bost, T., Denys, D., \& Vulink, N. (2020). Misophonia: Phenomenology, comorbidity and demographics in a large sample. PloS one, 15(4), e0231390.

Jager, I. J., Vulink, N. C., Bergfeld, I. O., van Loon, A. J., \& Denys, D. A. (2020). Cognitive behavioral therapy for misophonia: A randomized clinical trial. Depression and Anxiety.

Jastreboff, M. M., \& Jastreboff, P. J. (2002). Decreased sound tolerance and tinnitus retraining therapy (TRT). Australian and New Zealand Journal of Audiology, 24(2), 74-84. doi:10.1375/audi.24.2.74.3110

Jastreboff, P. J., \& Jastreboff, M. M. (2014). Treatments for decreased sound tolerance (hyperacusis and misophonia). Seminars in Hearing, 35(2), 105-120 https://doi.org/10.1055/s-0034-1372527

Kamody, R. C., \& Del Conte, G. C. (2017). Using dialectical behavior therapy to treat misophonia in adolescence. The Primary Care Companion for CNS Disorders, 19(5), https://doi.org/10.4088/pcc.17l02105

Kumar, S., Tansley-Hancock, O., Sedley, W., Winston, J. S., Callaghan, M. F., Allen, M., ... \& Griffiths, T. D. (2017). The brain basis for misophonia. Current Biology, 27(4), 527-533. doi: 10.1016/j.cub.2016.12.048

Lehrer, P. M., Woolfolk, R. L., Rooney, A. J., McCann, B., \& Carrington, P. (1983). Progressive relaxation and meditation: A study of psychophysiological and therapeutic differences between two techniques. Behaviour Research and Therapy, 21(6), 651-662. doi:10.1016/00057967(83)90083-9

Li, Y., Wang, R., Tang, J., Chen, C., Tan, L., Wu, Z., .. \& Wang, X. (2015). Progressive muscle relaxation improves anxiety and depression of pulmonary arterial hypertension patients. Evidence-based complementary and alternative medicine, 2015.

Lolak, S., Connors, G. L., Sheridan, M. J., \& Wise, T. N. (2008). Effects of progressive muscle relaxation training on anxiety and depression in patients enrolled in an outpatient pulmonary rehabilitation program. Psychotherapy and psychosomatics, 77(2), 119-125.

McGuire, J. F., Wu, M. S., \& Storch, E. A. (2015). Cognitive-behavioral therapy for 2 youths with misophonia. Journal of Clinical Psychiatry, 76(5), 573-574. https://doi.org/10.4088/JCP.14cr09343

McKay, D., Kim, S. K., Mancusi, L., Storch, E. A., \& Spankovich, C. (2018). Profile analysis of psychological symptoms associated with misophonia: a community sample. Behavior therapy, 49(2), 286-294. 
Öst, L. G. (1987). Applied relaxation: description of a coping technique and review of controlled studies. Behaviour Research and Therapy, 25(5), 397-409. doi:10.1016/0005-7967(87)90017-9

Öst, L. G. (1988). Applied relaxation: Description of an effective coping technique. Cognitive Behaviour Therapy, 17(2), 83-96. doi:10.1016/0005-7967(87)90017-9

Palumbo, D. B., Alsalman, O., De Ridder, D., Song, J. J., \& Vanneste, S. (2018). Misophonia and potential underlying mechanisms: a perspective. Frontiers in psychology, 9, 953.

Potgieter, I., MacDonald, C., Partridge, L., Cima, R., Sheldrake, J., \& Hoare, D. J. (2019). Misophonia: A scoping review of research. Journal of clinical psychology, 75(7), 1203-1218.

Quek, T. C., Ho, C. S., Choo, C. C., Nguyen, L. H., Tran, B. X., \& Ho, R. C. (2018). Misophonia in Singaporean psychiatric patients: a cross-sectional study. International journal of environmental research and public health, 15(7), 1410.

Reid, A. M., Guzick, A. G., Gernand, A., \& Olsen, B. (2016). Intensive cognitive-behavioral therapy for comorbid misophonic and obsessive-compulsive symptoms: A systematic case study. Journal of Obsessive-Compulsive and Related Disorders, 10, 1-9.

Roushani, K., \& Honarmand, M. M. (2021). The Effectiveness of Cognitive-behavioral Therapy on Anger in Female Students with Misophonia: A Single-Case Study. Iranian Journal of Medical Sciences, 46(1), 61.

Rouw, R., \& Erfanian, M. (2018). A large-scale study of misophonia. Journal of clinical psychology, 74(3), 453-479.

Schneider, R. L., \& Arch, J. J. (2017). Case study: A novel application of mindfulness- and acceptancebased components to treat misophonia. Journal of Contextual Behavioral Science, 6, 221-225. https://doi.org/10.1016/j.jcbs.2017.04.003

Schröder, A., van Wingen, G., Eijsker, N., San Giorgi, R., Vulink, N. C., Turbyne, C., \& Denys, D. (2019). Misophonia is associated with altered brain activity in the auditory cortex and salience network. Scientific reports, 9(1), 1-9.

Schröder, A., Vulink, N., \& Denys, S. (2013). Misophonia: Diagnostic criteria for a new psychiatric disorder. PLOS ONE 8: e54706.

Schröder, A. E., Vulink, N. C., van Look, A. J., \& Denys, D.A. (2017). Cognitive behavioral therapy is effective in misophonia: An open trial. Journal of Affective Disorders, 217, 289-294. https://doi.org/10.1016/j.jad.2017.04.017

Siepsiak, M., Sobczak, A. M., Bohaterewicz, B., Cichocki, Ł., \& Dragan, W. Ł. (2020). Prevalence of Misophonia and Correlates of Its Symptoms among Inpatients with Depression. International journal of environmental research and public health, 17(15), 5464.

Swedo, S., Baguley, D. M., Denys, D., Dixon, L. J., Erfanian, M., Fioretti, A., ... \& Raver, S. M. (2021). A Consensus Definition of Misophonia: Using a Delphi Process to Reach Expert Agreement. medRxiv. doi: https://doi.org/10.1101/2021.04.05.21254951

Taylor, S. (2017). Misophonia: A new mental disorder?. Medical Hypotheses, 103, 109-117.

Ulrich, R. E., \& Azrin, N. H. (1962). Reflexive fighting in response to aversive stimulation. Journal of the Experimental Analysis of Behavior, 5, 511-520. doi: 10.1901/jeab.1962.5-511

Yılmaz, Y., \& Hocaoğlu, Ç. (2021). Misophonia: A Review. Current Approaches in Psychiatry/Psikiyatride Guncel Yaklasimlar, 13(2).

Wu, M. S., Lewin, A. B., Murphy, T. K. \& Storch, E. A. (2014), Misophonia: Incidence, phenomenology, and clinical correlates in an undergraduate student sample. Journal of Clinical Psychology. Vol. 0O(00), 1-14. doi: 10.1002/jclp.220984a.

Zald, D. H., Lee, J. T., Fluegel, K. W., \& Pardo, J. V. (1998). Aversive gustatory stimulation activates limbic circuits in humans. Brain, 121(6), 1143-1154. doi:

http://dx.doi.org/10.1093/brain/121.6.1143 
Zald, D. H., \& Pardo, J. V. (1997). Emotion, olfaction, and the human amygdala: Amygdala activation during aversive olfactory stimulation. Proceedings of the National Academy of Sciences, 9(8), 4119-24.

\section{Supplementary Materials}

The notes from this case file are available for review. All references to identifying details of the client were removed. 